\title{
USO DO CANABIDIOL OU DA DIETA CETOGÊNICA NAS EPILEPSIAS REFRATÁRIAS: UMA BREVE REVISÃO DE LITERATURA.
}

\author{
CANNABIDIOL OR KETOGENIC DIET FOR REFRACTORY EPILEPSIES: A BRIEF LITERATURE REVIEW
}

Tamiris Carneiro Mariano, Juliana Pastana Ramos de-Freitas, Sebastião Gilberto Mota Tavares-Júnior, Laianne Barros Martins de Alcântara,

Pedro Henrique Daldegan Couto, Jeanne Alves de Sousa Mazza, Lisiane Seguti Ferreira

DOI - 10.5935/2236-5117.2021v58a66

\section{RESUMO}

O uso do canabidiol e da dieta cetogênica como tratamento para epilepsias refratárias tem ganhado atenção considerável, principalmente devido ao aumento de pesquisas sobreesses temas. Osefeitos medicinais daplanta Cannabis sativa, de onde é retirado o canabidiol (CBD), são conhecidos e aplicados há milhares de anos, com relatos do seu uso para tratamento de diversas condições médicas datando de 2700 a.C na China. Há relatos do uso da dieta cetogênica para controle da epilepsia por povos no passado. Atualmente a dieta está disponível em mais de 50 países. Entretanto, ambas as terapêuticas possuem baixa aceitação por pacientes e seus familiares e por médicos especialistas, o que pode estar relacionado ao desconhecimento, ao preconceito e aos sacrifícios necessários para a efetividade do tratamento. Desta forma, é importante a desmistificação do uso medicamentoso do canabidiol e da dieta cetogênica, principalmente pelos médicos neurologistas, que devem buscar aperfeiçoamento constante para oferecerem o melhor tratamento a seus pacientes. Uma boa relação médico-paciente deve esclarecer os benefícios desses tratamentos e promover maior aceitabilidade pelos pacientes e seus familiares e eficiência a longo prazo das terapias. 0 presente estudo traz uma revisão sobre 0 histórico, estudos clínicos, mecanismo de ação e efeitos adversos do canabidiol e da dieta cetogênica, além da apresentação e tolerabilidade, interações medicamentosas e vulnerabilidade do canabidiol, e os tipos de dieta, indicações e contraindicações, seguimento e exames complementares da dieta cetogênica para melhor informar os profissionais de saúde sobre essas possibilidades muitas vezes esquecidas de controle das epilepsias refratárias.

Palavras-chave: Canabidiol. Dieta Cetogênica. Epilepsia

\section{ABSTRACT}

Introduction: Text The use of cannabidiol and the ketogenic diet as a treatment for refractory epilepsies has gained considerable attention, mainly due to increased research on these topics. The medicinal effects of the Cannabis sativa plant, from which cannabidiol (CBD) is extracted, have been known and applied for years, with reports of its use for treating various medical conditions dating from 2700 BC in China. There are reports of the utilization of the Ketogenic Diet to control epilepsy by people in the past. The diet is now
Tamiris Carneiro Mariano - Hospital Universitário de Brasília, Medicina da Criança e do Adolescente - Brasília - Distrito Federal - Brasil.

Juliana Pastana Ramos de-Freitas - Hospital Universitário de Brasília, Medicina da Criança e do Adolescente - Brasília - Distrito Federal - Brasil.

Sebastião Gilberto Mota Tavares-Júnior-Universidade de Brasília, Faculdade de Medicina - Brasília - Distrito Federal - Brasil.

Laiane Barros Martins de Alcântara - Universidade de Brasília, Faculdade de Medicina - Brasília - Distrito Federal - Brasil.

Pedro Henrique Daldegan Couto - Universidade de Brasília, Faculdade de Medicina - Brasília - Distrito Federal - Brasil.

Jeanne Alves de Sousa Mazza - Hospital Universitário de Brasília, Medicina da Criança e do Adolescente - Brasília - Distrito Federal - Brasil.

Lisiane Seguti Ferreira - Hospital Universitário de Brasília, Medicina da Criança e do Adolescente - Brasília - Distrito Federal - BrasilUniversidade de Brasilia, Faculdade de Medicina - Brasília - Distrito Federal - Brasil.

Correspondência: Lisiane Seguti Ferreira - SGAN, Quadra 604,
Avenue L2 Norte, s/n - CEP: 70840- 050. Asa Norte. Brasília-DF Brasil

Internet: lisianeseguti@gmail.com

Conflito de interesses: não existem conflitos de interesse.

available in more than 50 countries. However, both therapies have low acceptance by patients, their relatives, and specialist physicians, which can be related to ignorance, prejudice, and the sacrifices needed for the effectiveness of treatment. Thus, it is crucial to demystify the drug use of cannabidiol and the ketogenic diet, mainly by neurologists, who must seek constant improvement to offer the best treatment to their patients. An effective doctor-patient relationship should clarify the benefits of these treatments and provide greater acceptability by the patient and his or her relatives and long-term efficiency of therapies. The present study provides a review of the history, clinical studies, mechanisms of action, and adverse effects of cannabidiol and the ketogenic diet, in addition to the presentation and tolerability, drug interactions and vulnerability of cannabidiol and the types of diet, indications, and contraindications, followup and complementary tests of the ketogenic diet to better inform health professionals about these often overlooked possibilities of controlling refractory epilepsies.

Keywords: Cannabidiol. Diet, Ketogenic. Epilepsy 


\section{INTRODUÇÃO}

A dieta cetogênica (DC) e o canabidiol (CBD) vem sendo cada dia mais utilizados na medicina, entretanto suas aplicações passam por muitos desafios de aceitação tanto do público leigo, quanto dos profissionais que desconhecem suas aplicabilidades e manejos clínicos para o tratamento de algumas patologias como, por exemplo, em casos de epilepsias refratárias.

A Cannabissp. apresenta grande diversidade de espécies, as quais possuem ao menos 545 compostos conhecidos 1. Dentre estes, o canabidiol (CBD) e o tetrahidrocanabinol (THC) são as principais, sendo os princípios ativos mais utilizados nos produtos derivados da Cannabis sp. cujo termo "cannabis medicinal" denomina os uma série de produtos com composição quantitativa e qualitativa diferentes de CBD e THC ${ }^{2}$.

Substâncias naturais ou artificiais que ativam receptores canabinóides são genericamente denominados canabinóides. Tais compostos estão envolvidos no sistema endocanabinóide ou canabinóide endógeno, responsável pela regulação de uma gama de mecanismos fisiológicos e cognitivos, além de ser um alvo significativo para o controle de crises epilépticas ${ }^{1}$. Em síndromes como a de Dravet e a de Lennox Gastaut, a liberação do CBD para controle de crises epilépticas de difícil controle, acabou por trazer benefícios não apenas no manejo da epilepsia, mas extensivo a condições como dor neuropática, espasticidade em doenças degenerativas, dentre outras ${ }^{3}$.

Muitas são as formas legalizadas de CBD, que podem ser adquiridas, inclusive no formato on-line nos Estados Unidos 4 . Algumas famílias, sem acesso à forma industrializada, optam pela produção caseira, de substância potencialmente ineficaz, sem qualquer cuidado no preparo, além da exposição aos riscos de sub e sobre dosagem e aos vários efeitos adversos, que incluem sonolência excessiva e transtornos gastrintestinais, dentre outros. Algumas interações medicamentosas já bem documentadas compreendem o ácido valpróico e o clobazam, os quais, respectivamente, aumentam a hepatotoxicidade, a sonolência e a produção de muco, podendo acarretar em maior risco de contaminação por infecções respiratórias ${ }^{2}$.

Outro tratamento médico eficaz para as crises epiléticas é a Dieta Cetogênica (DC), cujos registros de uso datam do início do século XX, mais precisamente em 1921, quando foi cunhado o termo dieta cetogênica pelo $\mathrm{Dr}$. Russell Wilder. No final do século $X X$, houve um avanço e uma aplicação intensiva do método, que se estabeleceu como tratamento comprovadamente eficaz para o manejo da epilepsia ${ }^{5}$. Desenvolvida para mimetizar os efeitos fisiológicos do jejum, a DC possui baixo índice glicêmico, equilíbrio proteico e é rica em gordura, cujas proporções exercem efeito neuro-protetor, acarretando controle das crises ${ }^{6}$.

Algumas variantes da DC, como a Dieta de Atkins modificada (DAM) e a Dieta de baixo índice glicêmico (DBIG), foram desenvolvidas a fim de torná-la mais palatável, favorecendo a aderência, e reduzir os efeitos adversos, sendo menos restritivas que a Dieta Cetogênica Clássica (DCC), mas com composição nutritiva semelhante ${ }^{6}$.

Nos casos em que haja falha do tratamento com, pelo menos, dois fármacos anti-crise, prescritos de forma correta em mono ou politerapia, a DC deve ser considerada ${ }^{7}$. A prescrição deve ser acompanhada em conjunto pelo neurologista e nutricionista do paciente e feita após avaliação médica e a realização dos exames complementares $^{8}$.

Este artigo traz uma breve revisão atualizada, acerca de dois tratamentos alternativos para epilepsia refratária, como o canabidiol e a dieta cetogênica. Serão abordados aspectos como dados históricos, mecanismos de ação e resposta terapêutica, dentre outros.

\section{MÉTODOS E RESULTADOS}

A elaboração desta revisão ocorreu em duas etapas, detalhadas a seguir: busca e seleção.

\section{Busca}

Foram realizadas buscas nas seguintes bases de dados: LILACS, MEDLINE via Pubmed e SciELO por meio dos descritores: ("Canabidiol" OU "Cannabis") AND "Epilepsy" AND ("Treatment" OR "Therapeutics"); "Ketogenic diet" AND "Epilepsy" AND ("Treatment" OR "Therapeutics"); associados a títulos, resumos ou palavras-chave. PubMed/National Library of Medicine (NLM), Cochrane Library, publicados no período de Janeiro/2005 a Dezembro/2019.

\section{Seleção}

A triagem foi realizada em um primeiro momento, por meio da avaliação dos resumos resultantes das buscas nas bases de dados. Procedeu-se em seguida à leitura completa dos artigos assim separados, com análise de 
impacto e de qualidade dos estudos. Foram incluídas publicações em língua inglesa e portuguesa.

\section{Síntese dos dados}

Para o estudo, foram levantadas 65 referências, das quais 40 associam-se ao tema canabidiol e epilepsia e 25 à dieta cetogênica e epilepsia, publicados principalmente em revistas indexadas. No entanto, optou-se por excluir 15 delas e restaram 45 referências, que julgamos pertinentes. A revisão será apresentada em tópicos: 1) Canabidiol e Epilepsia refratária: histórico, mecanismo de ação, estudos clínicos, apresentação e tolerabilidade, efeitos adversos, interações medicamentosas, vulnerabilidade; 2) Dieta Cetogênica e epilepsia refratária: histórico, mecanismo de ação, estudos clínicos, tipos de dieta, indicações e contraindicações, seguimento, exames complementares e efeitos adversos.

\section{CANABIDIOL E EPILEPSIA REFRATÁRIA}

\section{Histórico}

A planta Cannabis sativa é a terceira droga recreacional mais consumida no mundo, utilizada por cerca de $4 \%$ da população ${ }^{~}$. Seus efeitos medicinais são conhecidos e aplicados há milhares de anos. Há relatos com indicações do uso da planta na China datando de 2.700 a. C. para tratamento de diversas condições médicas como constipação intestinal, dores, malária, expectoração, epilepsia, tuberculose, dentre outras. Na Índia, há registros do uso da planta em $1.000 \mathrm{~A}$. C., administrada como hipnótico e ansiolítico para tratamento de ansiedade, manias e histeria ${ }^{10}$.

Já no início do século $X X$, comercializavam-se extratos de Cannabis na Inglaterra, Alemanha e Estados Unidos (EUA) para o tratamento de transtornos mentais, especialmente na função de hipnóticos e sedativos. $N a$ terceira década do século $X X$, houve uma redução no uso de Cannabis para fins médicos, o que pode ser atribuído a introdução do fenobarbital, em 1912, e da fenitoína, em 1937, além da aprovação do imposto sobre a Cannabis (Marijuana Tax Act) em 1937. (1). A partir de 1960, notou-se incremento significativo no uso recreativo da Cannabis, principalmente entre os jovens do mundo ocidental ${ }^{10}$.

Em 1964, o grupo do professor Raphael Mechoulam, israelense, isolou os principais componentes da planta Cannabis sativa e identificou as suas respectivas estruturas químicas. Inicialmente, o composto que recebeu a maior atenção dos pesquisadores foi o delta 9-tetraidrocanabidiol (THC) por ser responsável pelos efeitos psicoativos da planta ${ }^{1}$.
Na década de 1990, a descoberta de um sistema endógeno de sinalização de canabinóides reacendeu o interesse em terapias derivadas de constituintes da cannabis para distúrbios do sistema nervoso, como a epilepsia ${ }^{1}$.

Nos últimos anos, o apelo para liberação do CBD na mídia cresceu movido principalmente por pais de pacientes com epilepsia. No dia 20 de abril de 2018, Chuck Schumer, senador do estado de Nova York pelo partido democrata, introduziu uma legislação para descriminalizar a maconha nacionalmente, dando um grande passo na trajetória da aprovação do CBD nos EUA ${ }^{11}$.

O CBD foi aprovado nos EUA em junho de 2018 pela agência federal U.S Food and Drug Administration (FDA) como medicamento anti-crise adicional em pacientes a partir dos dois anos de idade que apresentam síndrome de Dravet ou de Lennox-Gastaut. Assim o Epidiolex®, fabricado pela empresa farmacêutica britânica GW Pharma, foi a primeira formulação industrializada a ser liberada oficialmente nos EUA ${ }^{11}$.

Atualmente o uso de cannabis medicinal é autorizado em quase todos os estados dos EUA e, pelo menos, em outros 40 países ${ }^{3}$. Nos locais onde o uso é permitido, existe uma grande variação nas leis que vão desde a rigorosa descriminalização e liberação para tratamento médico cuidadosamente regulamentado, até a completa legalização, no Canadá, Uruguai e alguns estados dos EUA ${ }^{12}$.

No Brasil, a Agência Nacional de Vigilância Sanitária (Anvisa) disponibiliza o serviço de autorização para importação excepcional de produtos à base de Canabidiol, em associação com outros canabinoides. 0 pedido de autorização deve ser feito por uma pessoa física, para uso próprio, mediante prescrição de profissional médico especialista legalmente habilitado, para tratamento de saúde, com laudo médico e termo de responsabilidade. A autorização da Anvisa é requisito obrigatório para importar esse produto ${ }^{13}$.

No dia 3 de dezembro de 2019, a Anvisa aprovou o registro de medicamentos à base de cannabis no Brasil. A medida entrou em vigor após 90 dias da publicação no Diário Oficial da União. A norma não é definitiva e deverá ser revista a cada 3 anos ${ }^{14}$.

\section{Mecanismo de ação}

O mecanismo de ação dos canabinoides foi elucidado com a descoberta de dois receptores endocanabinoides denominados CB1 (receptor canabinoide tipo 1) e CB2 (receptor canabinoide tipo 2). Os efeitos farmacológicos 
dos canabinoides são provenientes da interação destes com os receptores endocanabinoides. Os receptores CB1 são amplamente distribuídos no organismo e encontrados sobretudo pré-sinapticamente no sistema nervoso central em áreas ligadas ao controle motor, aprendizagem, memória, cognição e emoção, além de serem responsáveis pela maioria dos efeitos psicotrópicos dos canabinoides. Os receptores CB2 localizam-se principalmente no sistema imunológico e em áreas específicas do sistema nervoso central, como a micróglia e na região pós- sináptica. Podem estar associados à regulação da liberação de citocinas provenientes de células imunitárias e da migração destas, atenuando a inflamação e alguns tipos de dor ${ }^{15}$.

Os ligantes endógenos dos receptores $\mathrm{CB} 1$ e $\mathrm{CB} 2$ são denominados endocanabinoides e desempenham papel importante na modulação de neurotransmissão, especialmente como transmissores retrógrados na maioria dos processos fisiológicos, incluindo a dor, a cognição, a regulação do sistema endócrino, da função metabólica, resposta emocional e processos motivacionais. Os principais agonistas endógenos dos receptores endocanabinoides são os derivados do ácido araquidônico, constituídos de natureza lipídica assim como os canabinoides presentes na Cannabis sativa ${ }^{16}$.

Em condições fisiológicas normais, a ativação dos receptores endocanabinoides promove a alteração de vários neurotransmissores, incluindo a acetilcolina, a dopamina, o ácido gama amino-butírico (GABA), o glutamato, a serotonina, a noradrenalina e opioides endógenos ${ }^{15}$. Embora o mecanismo de ação do CBD não esteja totalmente elucidado, é provável que ele interaja com receptores específicos, tal como o $\Delta$-THC. $O$ CBD possui a habilidade de facilitar a sinalização dos endocanabinoides por intermédio do bloqueio da recaptação ou hidrólise enzimática da anandamida. No entanto, ele apresenta baixa afinidade pelos receptores CB1 e CB2. Porém, sugere-se que a capacidade do CBD de ampliar a biodisponibilidade da anandamida esteja relacionada com sua ação antipsicótica ${ }^{17}$.

O CBD atua em vários locais, incluindo alvos intracelulares, como mitocôndrias, e alvos localizados nos canais de membranas neuronais (canais de sódio dependentes de voltagem, canais de cálcio dependentes de voltagem), receptores de neurotransmissores (GABA, 5 -HT) e receptores acoplados à proteína G (GPR55) ${ }^{18}$.

0 receptor $\mathrm{CB} 1$ é o local onde o THC atua, enquanto o $C B 2$ é o alvo do canabinol. Existe a hipótese de que o $\mathrm{CBD}$ tenha efeitos neuroprotetores e anti-inflamatórios, os quais seriam características interessantes em um medicamento antiepiléptico ${ }^{18}$.

\section{Estudos clínicos}

Nos últimos anos, os estudos de CBD realizados por Devinksy et al. estabeleceram o benefício do Epidiolex $®$ nas síndromes de Dravet e de Lennox-Gastaut. 0 estudo da síndrome de Dravet recrutou 120 crianças e adultos jovens, enquanto os artigos abordando Lennox - Gastaut randomizaram 225 crianças e 171 adultos jovens. 0 resultado encontrado foi que $5 \%$ dos pacientes com síndrome de Dravet tratados com o medicamento ficaram totalmente livres de crises. Os dois documentos sobre a síndrome de Lennox-Gastaut analisaram as crises de queda ("drop attacks"), tipo de crise mais grave e incapacitante nos pacientes com essa condição. 0 estudo foi randomizado em três grupos, permitindo a comparação de doses de 10 e $20 \mathrm{mg} / \mathrm{Kg} / \mathrm{dia}$. Todos os grupos mostraram uma redução significativa nas crises de queda em comparação com o placebo ${ }^{19,20}$.

Uma revisão abrangente, reunindo ensaios clínicos randomizados e estudos abertos, descreveu resultados menos promissores. Estimou ser necessário tratar 8,3 indivíduos para 1 ter uma redução superior a 50\% nas crises convulsivas. Apenas 1 a cada 171 ficaram livres de crises ${ }^{21}$.

Uma metanálise brasileira recente analisou 11 estudos clínicos observacionais, publicados em revistas de grande impacto, sobre o tratamento da epilepsia refratária com produtos à base de canabidiol. Dois terços dos pacientes relataram melhora na frequência de crises epilépticas. Destes, a maioria (71\%) fazia uso de produtos com extratos ricos em CBD, com o restante usando CBD purificado, sendo esse resultado estatisticamente significante. Apenas 39\% dos pacientes apresentaram uma redução de $50 \%$ ou mais na frequência de crises epilépticas e não houve diferença entre tratamentos com extratos ricos em CBD e o CBD puro em relação à eficácia, entretanto a dose usada no CBD puro foi até quatro vezes maior que a do CBD com outros componentes da planta. Encontraram-se ainda mais efeitos adversos nos usuários de CBD purificado, que provavelmente está relacionada com a dose que necessita ser maior para atingir o controle das crises ${ }^{22}$

A interpretação de maior potência de CBD em combinação com outros compostos menores está de acordo com outros estudos que indicam efeitos sinérgicos entre canabinoides e até mesmo compostos não canabinoides presentes na planta. 
Outros compostos, como a canabidivivarina (CBDV), tetrahidrocanabivivarina (THCV) e canabinol (CBN) também possuem ação anticrise.

Outra interação que vem sendo estudada é o uso do CBD comTHC, emostrou-se que o CBD tende abloquear alguns dos eventos adversos do THC, como ansiedade e psicose. A farmacologia sugere que esses efeitos são devidos à modulação alostérica de receptores canabinoides $\mathrm{CB} 1$, que atuam refinando o receptor canabinoide CB1, afetando a interação de outros canabinóides no nível do receptor. O THC se liga diretamente aos receptores CB1 e $C B 2$, mantendo uma maior afinidade pelos receptores CB1. O CBD tem pouca afinidade pelos dois receptores canabinóides. Em vez disso, atua como um antagonista indireto dos agonistas canabinóides. O CBD liga-se muito fracamente aos receptores CB1 e necessita do THC para permitir essa ligação e auxiliar a reduzir os efeitos psicoativos do THC, tais como euforia e sedação. Entretanto, para o uso em crianças com epilepsia de difícil controle, a recomendação continua sendo o uso do CBD purificado, devido à interferência do THC no processo de poda neuronal. São necessários estudos adicionais para se avaliar a eficáciae a posologia mais adequada do CBD puro e das formulações, que contém THC. ${ }^{22}$

\section{Apresentação e tolerabilidade}

As drogas farmacêuticas disponíveis são purificadas apenas com CBD, ou formulações contendo THC, conhecidas como full spectrum. Quanto mais THC o medicamento contém, mais psicoativo ele outros extratos sem o THC, conhecida como broad spectrum, ou será, o que torna imperativo ajustar as doses equivalentes de $C B D$ e THC para minimizar os efeitos contendo $C B D$, outros extratos e o deletérios deste último composto.

Esse ajuste tem sido relativamente equalizado com as preparações da Indústria Farmacêutica, diferentemente das apresentações caseiras/artesanais, cuja eficácia e efeitos adversos são imprevisíveis. Usualmente, os produtos são bem tolerados e os efeitos colaterais sobressalentes são os gastrointestinais ${ }^{23}$

A biodisponibilidade oral do CBD é inferior a $10 \%$, e, devido à sua alta solubilidade lipídica, necessita de um veículo que permita sua administração em solução aquosa. $\mathrm{OCBD}$ pode ser encontrado nas formas de pasta, suspensos em óleo, álcool ou líquido (spray). Atinge níveis séricos máximos dentro de $90-120$ minutos e possui uma meia vida de 18 a 32 horas ${ }^{23,4}$.
Outras formulações (intranasal, transdérmica, transmucosa) já estão disponibilizadas. Os níveis séricos de CBD oral e seus metabólitos aumentam proporcionalmente com a dose. Para crianças com epilepsia refratária, o recomendado é o CBD puro, com aumento lento e gradual da dose. 0 Epidiolex® TM foi a primeira formulação de CBD puro, a ser autorizado no Brasil e licenciado paradoses entre 2,5 até $20 \mathrm{mg} / \mathrm{kg} / \mathrm{dia}$ (geralmente dividido em duas doses). Doses maiores muitas vezes podem ser necessárias, especialmente no caso do CBD puro; no entanto, são limitadas pela baixa tolerabilidade ${ }^{20}$.

\section{Efeitos adversos}

Eventos adversos comuns são, principalmente, diarreia, apetite reduzido, vômito, alteração da função hepática e sonolência. Geralmente são leves e melhoram com a redução da dose ${ }^{21}$.

Estado de mal epiléptico e morte súbita são relatados (5-10\%), mas nenhum até agora atribuído diretamente ao tratamento. Cerca de 2,2\% experimentam efeitos colaterais graves, com sonolência excessiva (risco de aspiração, dificuldades posturais, quedas), diarreia grave, ou elevação das enzimas hepáticas maior que três vezes o limite da normalidade ${ }^{3}$.

O FDA relatou maior taxa de infecção $(4 \%)$ dos pacientes em tratamento em relação ao placebo $(0 \%)$, com o risco de pneumonia notavelmente maior ( 9,1 vezes) naqueles que recebem $\mathrm{CBD}^{3}$.

\section{Interações medicamentosas}

Os fármacos com interações mais bem documentadas são o ácido valpróico e o Clobazam. O valproato aumenta o risco de hepatotoxicidade. O CBD inibe o citocromo P450 (CYP) 2C19 e aumenta as concentrações séricas do Clobazam, que eleva a taxa de infecções do trato respiratório, devido ao rebaixamento do sensório e aumento das secreções desses pacientes. Os efeitos podem ser controlados com ajuste de doses e seguimento rigoroso ${ }^{15}$.

\section{Vulnerabilidade}

Os neurologistas são constantemente procurados pelos pais dos pacientes com epilepsia de difícil controle com o desejo de experimentar canabinóides como tratamento. 0 processo para liberação é burocrático e rigoroso, e muitos pacientes não têm indicação embasada nos 
estudos para iniciar o tratamento. Os pais acabam se frustrando e na ânsia de tentar um novo tratamento para o filho, muitas vezes adquirem via internet, produtos artesanais com a composição totalmente incerta ${ }^{2}$.

Um estudo Australiano revelou que até $15 \%$ dos adultos e $13 \%$ dos pais de crianças com epilepsia relataram uso de produto de cannabis em algum momento. Outro estudo de seguimento de 41 famílias, encontrou que apenas $76 \%$ divulgaram isso para os seus médicos ${ }^{24}$.

Até o momento, não existe uma maneira de quantificar o que há nessas formulações. Por essa razão, é papel do neurologista reforçar para seus pacientes que apenas os produtos produzidos por indústrias farmacêuticas regulamentadas são seguros. Ademais, não se deve aconselharsobrefontes, dosesouesquemas. Éimportante manter um bom vínculo com o paciente, aumentando a suspeita do uso de formulações não padronizadas. Assim, o médico deve monitorar as concentrações séricas dos fármacos antiepilépticos usados pelo paciente e identificar alterações clinicamente relevantes ${ }^{3}$.

Outro ponto que necessita atenção é o papel da resposta ao placebo, que é alto nos pacientes com epilepsia e, em particular, em uso de canabis medicinal. Tal fato pode ser atribuído ao apelo da mídia somada à cultura de acreditar que um produto natural é mais seguro e mais eficaz. Os pais tendem a demorar a relatar efeitos colaterais negativos ${ }^{26}$. Há ainda os efeitos benéficos do CBD no humor, função social e outras comorbidades que levam o paciente a se sentir melhor, mesmo sem controle das crises ${ }^{26}$.

\section{DIETA CETOGÊNICA E EPILEPSIA REFRATÁRIA:}

\section{Histórico}

A DC é um tratamento alternativo, não medicamentoso, amplamente utilizado para controle de crises epilépticas. É uma dieta rica em gorduras, pobre em carboidratos e com quantidade adequada de proteínas, mimetizando os efeitos bioquímicos do jejum. Desde a era de Hipócrates (460 a.C. - 370 a.C.), conforme registro, o jejum é considerado um tratamento para epilepsia. Também foi documentado nos tempos bíblicos: o evangelho, segundo Marcos (9:14-29) descreve uma passagem em que Jesus cura um menino com epilepsia, por meio de "oração e jejum" 5.

Nos anos de 1920 a 1930, quando apenas os brometos e o fenobarbital estavam disponíveis como anticonvulsivantes, a dieta foi utilizada para controle da epilepsia com relato de bons resultados. No início da década, o Dr. Hugo Conklin, médico osteopático, supôs que o jejum episódico reduziria as toxinas provenientes do intestino (as quais ele acreditava que eram a origem da epilepsia) e descobriu que o jejum de crianças por até 25 dias produzia notável controle de crises de forma imediata ou a longo prazo. O Dr. Geyelin relatou, na American Medical Association Convention, sua boa experiência com o jejum como tratamento da epilepsia. Em 1921, Dr. Russel Wilder, na Clínica Mayo, foi o primeiro a publicar que os benefícios do jejum poderiam ser obtidos por outros meios, nascendo o termo "dieta cetogênica". Em 1924, Dr. Peterman, também na Clínica Mayo, tornou efetivo o cálculo da DC que até hoje é utilizado ${ }^{8}$.

Em 1938, quando a fenitoína foi descoberta, a atenção de médicos e pesquisadores voltou-se para os novos fármacos anticrise e a DC acabou esquecida, sendo utilizada de forma esporádica no Hospital John Hopkins a partir de 1970 5. A DC retorna para a mídia na década de 90 após um caso real ganhar repercussão: o filho do produtor de Hollywood - Jim Abrahams, apresentava crises epilépticas de muito difícil controle e teve uma melhora expressiva, em poucos dias, após ser submetido, no Hospital John Hopkins, ao tratamento com a DC. Tal fato estimulou o pai a criar a Charlie Foundation, a qual difunde informações sobre a DC para pais, bem como para médicos e nutricionistas. Além disso, ainda forneceu recursos para publicações e apoiou o primeiro estudo multicêntrico prospectivo sobre a eficácia da DC. Em 1997, Jim Abrahams dirige o filme "First Do no Harm" sobre a luta deCharlie contra a epilepsia ${ }^{27}$.

Nos últimos anos, houve uma explosão de publicações sobre a DC. Somente para exemplificar, no PubMed, esse número subiu de 6 a 8 por ano para mais de 200 publicações por ano e a DC tornou-se disponível em pelo menos 50 diferentes países 5 . Mesmo assim, continua sendo subutilizada, o que se deve à dificuldade de criação e manutenção de Centros para administração e seguimento adequado desses pacientes, além de os neurologistas ainda preferirem o uso de medicamentos, deixando a DC como última opção ${ }^{\text {. }}$.

\section{Mecanismo de ação}

Por muitos anos, a cetose foi o principal mecanismo de ação aceito para justificar a melhora expressiva das crises pelo uso da DC, porém muitos pesquisadores não a atribuem esse papel, sendo que os mecanismos de ação da DC ainda não estão completamente esclarecidos. Estudos feitos em modelos animais in vivo e modelos in vitro apresentam os prováveis mecanismos 
complexos da DC, que controlam as crises epilépticas, exercem efeito neuroprotetor e ocasionam diversas alterações metabólicas, podendo-se citar como as mais importantes: aumento dos corpos cetônicos no plasma (beta-hidroxibutirato, acetona e acetoacetato), o efeito sedativo destes, o grau de acidose, a desidratação parcial, a estabilização dos níveis séricos de glicose, efeito dos ácidos graxos poliinsaturados (PUFAs), aumento dos níveis de aminoácidos no LCR, mudanças na concentração lipídica e a adaptação do cérebro ${ }^{6,28,29}$.

Na dieta, o corpo utiliza a gordura como fonte de energia. Em uma dieta habitual, o acetil-CoA (obtido a partir da metabolização dos lipídios no fígado) segue para a via do ciclo de Krebs e produz energia, na forma de adenosina trifosfato (ATP). Na DC, devido à baixa concentração de oxaloacetato, a velocidade de oxidação por meio dessa via é reduzida, favorecendo a cetogênese, ou seja, a geração dos corpos cetônicos denominados acetoacetato, acetona e $\beta$-hidroxibutirato. Os corpos cetônicos atravessam a barreira hematoencefálica, por meio dos transportadores de ácido monocarboxílico, e entram no ciclo de Krebs, sendo o substrato energético do cérebro ${ }^{30}$.

Os tipos de ácidos graxos ofertados na dieta podem interferir de maneira diferente nas vias metabólicas, como apontam estudos mais recentes. Os PUFAs, especialmente o ômega-3 e o ácido docosa-hexanoico (DHA), têm propriedades antiepilépticas e podem apresentar maior eficácia clínica no controle das crises epilépticas quando comparados com os ácidos saturados ${ }^{31}$.

Outros estudos em modelos animais sugeriram efeito anti-inflamatório e maior controle de crises epilépticas a partir de uma mudança da composição da membrana celular que pode estar associada à ativação de receptores de proliferação de peroxissomos (PPARs) ${ }^{32}$.

De forma geral, o efeito da DC está associado especialmente à modulação dos neurotransmissores, potencial de membrana, canais de íons, mudanças na concentração de monoaminas e ação neuroprotetora ${ }^{33}$.

A principal via estudada e a de maior evidência científicaé a concentração de neurotransmissores. Estudos referem que os corpos cetônicos modulam neurotransmissores e influenciam suas concentrações, especialmente as dos principais neurotransmissores excitatórios e inibitórios (glutamato e ácido Y-aminobutírico GABA, respectivamente) ${ }^{34}$.

Dentro dessa modulação dosneurotransmissores, háduas hipóteses: a hipótese GABAérgica e a glutamatérgica.
A primeira acredita que os corpos cetônicos parecem influenciar o metabolismo do glutamato, favorecendo o aumento da concentração do GABA: o GABA é um intermediário do $\alpha$ - cetoglutarato sintetizado no ciclo de Krebs via glutamato convertido em GABA pela glutamato descarboxilase. Os corpos cetônicos parecem estimular a ação dessa enzima por um possível efeito na queda da concentração de aspartato, levando a um aumento da síntese de GABA, que tem função inibitória, resultando no controle das crises epilépticas ${ }^{6}$.

A segunda hipótese se baseia no fato da razão glutamatocetose modular as funções fisiológicas por meio dos transportadores vesiculares de glutamato (VGLUT). Dessa forma, os corpos cetônicos, especialmente o acetoacetato atuam na modulação de VGLUT1 e VGLUT2 e na regulação do $\mathrm{Cl}$, inibindo a liberação do glutamato ${ }^{35}$.

Outro mecanismo de efeito da DC estudado em modelos animais é a modulação das aminas biogênicas, especialmente norepinefrina e adenosina ${ }^{29}$.

A dieta promove um efeito neuroprotetor, mantendo os pacientes muitas vezes sem crises, mesmo após o término da dieta. Esse êxito está relacionado com a ação direta na mitocôndria, a qual tem uma função importante no mecanismo de controle das crises epilépticas pela geração de ATP, controle da apoptose, manutenção da homeostase de cálcio e produção e detoxificação de ROS, que são geradas intensamente na epilepsia, já que a doença está associada ao estresse oxidativo ${ }^{36}$.

\section{Estudos clínicos}

Na literatura, até 2008, encontravam-se apenas estudos prospectivoseretrospectivos, demonstrando aeficáciada dieta, sendo que as meta-análises e revisões descreviam a falta de ensaios clínicos randomizados controlados ${ }^{6}$.

Um grande passo na história da DC ocorreu em junho de 2008, quando o primeiro estudo multicêntrico randomizado controlado não duplo cego, foi publicado na Lancet Neurology por Neal et al. ${ }^{31}$, em que foi avaliado a DC em comparação com pacientes apenas em uso de fármacos anticrise.

Os resultados desse estudo mostraram uma redução de $38 \%$ na frequência das crises nas 54 crianças que fizeram a DC em relação as 49 crianças do grupo controle, sendo considerado estatisticamente significante. Nenhuma criança no grupo controle obteve diminuição de mais de $90 \%$ das crises, sendo cinco no grupo experimental com DC. Os autores também não observaram diferenças quanto à eficácia da DC clássica comparativamente com a dieta com triglicerídeos de cadeia média ${ }^{31}$. 
Em 2009, outro estudo randomizado controlado e duplo cego, feito no Hospital Johns Hopkins em pacientes com a Síndrome de Lennox-Gastaut, encontrou uma redução expressiva no número de crises epilépticas. Entretanto, os dois grupos entraram em cetose, pois o grupo que recebeu placebo também fez o mesmo jejum de 36 horas que o grupo da DC, o que acabou confundindo a interpretação dos resultados, que seriam melhores se o grupo que recebeu placebo não entrasse em cetose ${ }^{37}$.

Em estudos retrospectivos e prospectivos, a dieta modificada de Atikns leva à redução das crises com porcentagem muito semelhantes à DC clássica (DCC) 38. Uma revisão sistemática da Cochrane Database, publicada em 2016, analisou sete ensaios clínicos randomizados e encontrou resultados semelhantes aos primeiros estudos em relação ao percentual de redução e controle total das crises. Os efeitos adversos mais comuns foram as síndromes gastrointestinais, sendo o principal fator para os participantes desistirem dos estudos, além da não aceitação da dieta ${ }^{6}$.

\section{Tipos de Dieta}

Baseada na DCC foram criadas modalidades da dieta para tentar aumentar a aderência e palatabilidade. Outras opções são: Dieta de Atkins modificada (DAM); Dieta cetogênica com triglicerídeos de cadeia médica (DCTCM); Dieta de baixo índice glicêmico (DBIG) ${ }^{32}$.

A DCC tem alto teor de gordura, cerca de $90 \%$ do valor calórico total da dieta, composta principalmente por triglicerídeos de cadeia longa (TCL), baixo teor de carboidrato e adequado de proteínas, sendo o mínimo de $1 \mathrm{~g} / \mathrm{Kg} / \mathrm{dia}$. É estritamente individualizada, minimamente calculada, baseada nas necessidades energéticas de cada indivíduo. Todos os alimentos são pesados. A dieta é realizada nas proporções $3: 1$ ( $3 \mathrm{~g}$ de gordura para $1 \mathrm{~g}$ de carboidrato e proteína) e 4:1 (4g de gordura para $1 \mathrm{~g}$ de carboidrato e proteína). Essa proporção pode ser modificada de acordo com a resposta clínica e a cetose do indivíduo ${ }^{37}$.

A dieta com TCM possibilita uma menor ingestão de gorduras, poisos TCMsãoabsorvidosmaiseficientemente que os TCL e são mais cetogênicos. Assim, uma menor quantidade de gordura é necessária na DCTCM para se atingir a cetose, correspondendo entre $70 \%$ a $75 \%$ do valor calórico total proveniente de TCM e TCL, o que permite um aumento na quantidade de proteínas e carboidratos. $O$ cálculo da dieta é baseado na quantidade de energia obtida por meio do TCM. Na DCTCM original, $60 \%$ do valor calórico seriam obtidos pelo TCM, $10 \%$ por proteína, $15 \%$ a $19 \%$ de carboidratos e $11 \%$ a $15 \%$ de TCL. Essa alta quantidade de TCM provoca cólicas, diarreia e vômitos na maioria das crianças, por isso a quantidade de TCM deve ser adequada a cada indivíduo, de acordo com a resposta clínica e a cetose. O TCM deve ser dividido em todas as refeições ao longo do dia ${ }^{39}$.

A DAM foi criada em 2003, no Hospital Johns Hopkins, é menos restritiva, sendo livre a quantidade de calorias, gordura e proteína, mas a de carboidratos permanece restrita. Cerca de $60 \%$ do valor calórico total são obtidos pela gordura. 0 cálculo e treinamento são mais fáceis, permitindo mais flexibilidade e independência do paciente, com resultados após 2 a 4 semanas se for bem aplicada. A combinação com fórmulas cetogênicas no início do tratamento aumenta a relação cetogênica, beneficiando a cetose e o controle das crises epilépticas 40,38 .

A DBIG foi desenvolvida em 2002, com o objetivo de manter os níveis de glicemia estáveis e mais baixos, através do consumo exclusivo de carboidratos com índice glicêmico (IG) menor ou igual a

50. Na DBIG, é permitida a ingestão de $40 \mathrm{~g}-60 \mathrm{~g}$ de carboidratos ao dia, o que corresponde a aproximadamente $10 \%$ do valor calórico total e incentiva o consumo de gordura para a média de $60 \%$ do valor calórico total. Os pais recebem uma tabela com os alimentos que são de baixo IG. Os efeitos adversos são leves, como perda de peso, acidose, refluxo gastroesofágico e constipação. Na DBIG pode ou não ocorrer a cetose, sendo que se o controle das crises epilépticas não for obtido com a DBIG, pode ser feita a transição para a DCC ${ }^{41}$.

A escolha da dieta depende da avaliação médica e nutricional completas, além da análise do perfil biopsicossocial da criança e da família. Em crianças menores de 2 anos, foi observada, de forma significativa, uma resposta melhor com a DCC, quando comparada à DAM ${ }^{42}$.

Em crianças que recebem a dieta por gastrostomia, a melhor indicação é a DCC, na proporção 3:1 ou 4:1. Pode ser feita com alimentos caseiros processados ou com as fórmulas cetogênicas comerciais prontas, que facilitam a administração e evitam a contaminação. Para crianças em estado grave, internadas em unidades de terapia intensiva em estado de mal refratário, a DCC 4:1 deve ser introduzida, de preferência com período de jejum de $12 \mathrm{~h}$, para induzir a cetose mais rapidamente ${ }^{43}$.

\section{Indicações e contraindicações}

A DC apresenta resultados bons em qualquer idade, sendo que há algumas epilepsias e síndromes que ela 
apresenta um melhor resultado, como, por exemplo, nos espasmos infantis ${ }^{34}$

A DC é indicada para pacientes, adultos e crianças, com epilepsia de difícil controle, que frequentemente apresentem crises diárias ou semanais e que tenham falhado no tratamento com dois, ou três fármacos anticrise, corretamente indicados, tolerados e utilizados em doses adequadas em monoterapia ou politerapia ${ }^{7}$.

É o tratamento de eleição das crises que ocorrem em associação com a síndrome de deficiência de transportador de glicose tipo 1 (GLUT1) e deficiência de piruvato desidrogenase. Deve ser usada precocemente na Síndrome de Dravet, Síndrome de West, Síndrome de Doose, Síndrome de Rett, Landau-Kleffner, Síndrome de Lennox-Gastaut, complexo esclerose tuberosa e estado de mal epiléptico induzido por febre ${ }^{32}$.

Outras indicações descritas nos estudos são: cefaleia, demência de Alzheimer, doença de Parkinson, distúrbios do sono, tumor cerebral, Transtorno do Espectro Autista, dor neuropática e esclerose múltipla ${ }^{44}$.

Dentre as contraindicações absolutas estão: deficiência primária da carnitina, deficiência da carnitina- palmitoil transferase tipo I ou II, deficiência da carnitina translocase 4, defeitos da betaoxidação (deficiência da acildesidrogenase de cadeia média; deficiência da acildesidrogenase de cadeia longa; deficiência da acildesidrogenase de cadeia curta; deficiência da 3-hidroxiacil-CoA de cadeia longa; deficiência da 3-hidroxiacil-CoA de cadeia média), deficiência de piruvato carboxilase e porfiria ${ }^{32}$.

\section{Seguimento e exames complementares}

Anteriormente, a DC era iniciada com o paciente internado e após 12 horas de jejum. O manejo exclusivamente ambulatorial traz a vantagem da redução da ansiedade dos pais e da criança, além de diminuir os custos associados à hospitalização. 0 seguimento é feito a cada 1 ou 3 meses, quando a nutricionista ajusta a dieta e o médico faz o seguimento clínico checando crescimento, exames laboratoriais, efeitos adversos e a resposta ao tratamento. As crianças são mantidas em DC por cerca de 2 anos ${ }^{32}$.

A cetose é um parâmetro importante, que deve ser acompanhado rigorosamente nos primeiros meses de adoção da dieta cetogênica, pois permite comprovar que o organismo está produzindo a cetose esperada e verificar a adesão ao tratamento. Pode ser feita no sangue com um aparelho simples ou na urina através das fitas de cetonúria. A família deve ser treinada antes ${ }^{43}$.

Os exames complementares necessários para iniciar a dieta e avaliados no seguimento dos pacientes são: hemograma; glicemia; sódio; potássio; cálcio; magnésio; enzimas hepáticas; ureia e creatinina; ácido úrico; colesterol total e frações; triglicerídeos; proteínas totais e frações; Gasometria venosa; vitamina D; zinco; selênio; carnitina total e livre; nível sérico dos fármacos antiepilépticos; sumário de urina; relação cálciocreatinina; ultrassonografia renal; eletrocardiograma; ecocardiograma; e densitometria óssea ${ }^{32}$.

No seguimento, os exames são feitos a cada três meses no primeiro ano e depois a cada seis meses, ultrassonografia a cada seis meses, densitometria óssea uma vez ao ano. A suplementação de cálcio e complexo vitamínico é mandatória na DC 32 .

Estudos demonstram que cerca de $50-60 \%$ das crianças têm uma redução de pelo menos $50 \%$ das crises e $30 \%$ delas possuem uma diminuição superior a $90 \%$, além de muitos pacientes manterem a melhora obtida com a DC mesmo anos depois, o que é visto também na prática clínica ${ }^{6,34}$.

\section{Efeitos adversos}

No início da dieta, pode ocorrer hipoglicemia, constipação ou diarreia, sintomas de cetose excessiva com taquipneia e taquicardia, rubor facial, irritabilidade, vômitos, letargia e recusa alimentar ${ }^{32,37}$.

Efeitos adversos a longo prazo que podem ocorrer são: hiperlipidemia em até $60 \%$ dos pacientes, sendo que após 6 meses o colesterol costuma normalizar; nefrolitíase de 3 a $10 \%$, até $25 \%$ nos pacientes com mais de 6 meses de dieta, devido a hipercalciúria que a DC provoca e pode ser evitada com alcalinizantes orais; alterações gastrointestinais em $70 \%$ (efeito mais comum), principalmente refluxo gastroesofágico e constipação; em alguns estudos, foi observada uma menor velocidade de crescimento durante a $D C$, que parece estar mais relacionada com o nível de cetose ao invés do conteúdo proteico; e alterações ósseas como osteoporose e osteopenia ${ }^{37,45}$.

\section{CONCLUSÕES}

O canabidiol e a dieta cetogênica são alternativas viáveis para o tratamento de epilepsias refratárias e, por isso, deve ser de conhecimento dos médicos em geral, 
quanto à prescrição e indicação destes, a fim de oferecer o melhor tratamento para seus pacientes, respeitando as particularidades de cada caso. 0 domínio de novas terapêuticas faz-se fulcral no âmbito profissional para o desempenho consistente do dever médico.

Para tal, o especialista deve sempre prezar pelo respeito aos pacientes e aos seus familiares e pela boa relação médico-paciente, buscando sanar dúvidas acerca do tratamento, riscos e efeitos adversos. A decisão do recurso terapêutico deve ser compartilhada entre eles e autorizada pelos familiares dos pacientes menores de idade, aumentando as chances de permanência e sucesso do tratamento.

0 estímulo ao interesse e a pesquisa dessas modalidades terapêuticas deve ser fomentada para a área acadêmica cuja divulgação e explicação destas auxilia o contato e instiga a busca por conhecimento e aprimoramento científico.

Além da atualização constante das novas terapias por especialistas e da divulgação científica entre os acadêmicos é de extrema importância desmistificar o uso do canabidiol para a população. A diferença entre a maconha utilizada de forma recreativa e o canabidiol purificado nas indústrias farmacêuticas deve ser ensinada ao público leigo, a fim de que estes entendam que há diferença entre a produção caseira $\mathrm{e}$ a industrial. Desta forma, a boa relação médico-paciente também permite que o paciente não busque alternativas não prescritas pelo médico, promovendo a melhor adesão ao tratamento.

Além da resistência natural da sociedade na aceitação do uso terapêutico do CBD; a sua prescrição no Brasil, até o final de 2019, foi bastante limitada, devido à necessidade de importar o medicamento de outros países. Entretanto, a partir da liberação e registro de venda dos produtos à base de cannabis pela ANVISA, em dezembro de 2019, houve maior divulgação e alcance do uso do CBD, inclusive com formulações de menor custo, disponibilizadas por Associações Filantrópicas, para tratamento não apenas de epilepsia refratária, mas também para outras finalidades terapêuticas. Mais recentemente, no Brasil, além da liberação da importação de canabidiol de outras marcas estrangeiras, também foi aprovada pela ANVISA, uma marca brasileira com formulação oral de CBD puro, já disponível no serviço público, em farmácias de dispensação de alto custo. Importante, no entanto, ressaltar que a formulação, a dose ideal e a real eficácia para o tratamento de epilepsia refratária ainda não são consenso.
Como a DC exige um trabalho em equipe entre médico, nutricionista e família, além de acompanhamento rigoroso com vários profissionais, muitas vezes torna-se a última opção para médicos e pacientes. Uma barreira importante para a aceitação da dieta é a própria cultura alimentar associada a momentos de prazer. Além disso, principalmente para as crianças, o alimento é utilizado como um mecanismo de recompensa ou de celebração. As restrições impostas pela DC podem afetar sobremaneira a qualidade de vida e sensação de bemestar dos pacientes e de sua família, promovida pela ingestão de alimentos "saborosos", que fazem parte da rotina normal da criança.

De acordo com a revisão realizada, concluímos que, até o presente momento, os resultados obtidos para controle da epilepsia refratária com DC parecem mais promissores e duradouros que o tratamento à base de CBD. Entretanto, ressaltamos que o fomento a pesquisas no que tange ao uso de CBD no Brasil devem ser incentivadas a fim de se formar uma base de dados concretos sobre a eficácia real deste tipo de tratamento alternativo a nível nacional.

Enfim, cabe ao médico em geral, ter conhecimento básico sobre essas modalidades de tratamento para bem informar aos seus pacientes e não deixar que o manejo da epilepsia para grande parte da população, continue sob as sombras.

\section{REFERÊNCIAS}

1. Friedman D, Devinsky O. Cannabinoids in the Treatment of Epilepsy. N Engl J Med 2015 Sep 10; 373 (11):1048-58.

2. Cross $\mathrm{JH}$, Cock $\mathrm{H}$. A perspective on cannabinoids for treating epilepsy: Do they really change the landscape? Neuropharmacology. 2020 Jun 15;170:107861

3. Thomas RH, Cunningham MO. Cannabis and epilepsy. Pract Neurol. 2018 Dec;18(6):465-471.

4. Bonn-Miller MO, Loflin MJE, Thomas BF, Marcu JP, Hyke T, Vandrey R. Labeling Accuracy of Cannabidiol Extracts Sold Online. JAMA. 7 de novembro de 2017; 318 (17): 1708-1709.

5. Wheless JW. History of the ketogenic diet. Epilepsia. 2008 Nov;49 Suppl 8:3-5.

6. Martin K, Jackson CF, Levy RG, Cooper PN. Ketogenic diet and other dietary treatments for epilepsy. Cochrane Database Syst Rev. 2016 Feb 9;2:CD001903.

7. Thammongkol S, Vears DF, Bicknell-Royle J, Nation J, Draffin K, Stewart KG et al (2012) Efficacy of the ketogenic diet: which epilepsies respond? Epilepsia 53(3):55-59

8. Freeman JM, Kossoff EH. Ketosis and the ketogenic diet, 2010: advances in treating epilepsy and other disorders. Adv Pediatr. 2010;57(1):315-29.

9. Budney AJ, Sofis MJ, Borodovsky JT. An update on cannabis use disorder with comment on the impact of policy related to therapeutic and recreational cannabis use. Eur Arch Psychiatry Clin Neurosci. 2019 Feb;269(1):73-86. 
10. Zuardi AW. History of cannabis as a medicine: a review. Braz J Psychiatry. 2006 Jun;28(2):153-7.

11. Dyer O. US authorities recommend first approval of cannabis derived drug. BMJ. 2018 Apr 23;361:k1794.

12. Hall W. The future of the international drug control system and national drug prohibitions. Addiction. 2018 Jul;113(7):1210-1223.

13. Brucki SM, Frota NA, Schestatsky P, Souza AH, Carvalho NV, Manreza ML et al. [Cannabinoids in neurology--Brazilian Academy of Neurology]. Arquivos de Neuro- psiquiatria. 2015 Apr;73(4):371-374.

14. Brasil. Ministério da Saúde. Agência Nacional de Vigilância Sanitária. Resolução da diretoria colegiada-RDC n० 325, de 03 de dezembro de 2019. Disponível em: < https://www. in.gov.br/web/dou/-/resolucao-da-diretoria-colegiadardc-n-325-de-3-de- dezembro-de-2019-231272961 > Acessado em 4 des 2019.

15. De Caro C, Leo A, Citraro R, De Sarro C, Russo R, Calignano $A$, Russo $E$. The potential role of cannabinoids in epilepsy treatment. Expert Rev Neurother. 2017 Nov;17(11):10691079.

16. Saito VM, Wotjak CT, Moreira FA. Exploração farmacológica do sistema endocanabinoide: novas perspectivas para o tratamento de transtornos de ansiedade e depressão? [Pharmacological exploitation of the endocannabinoid system: new perspectives for the treatment of depression and anxiety disorders?]. Braz J Psychiatry. 2010 May;32 Suppl 1:S7-14. Portuguese.

17. Schubart CD, Sommer IE, Fusar-Poli P, de Witte L, Kahn RS, Boks MP. Cannabidiolas a potential treatment for psychosis. Eur Neuropsychopharmacol. 2014 Jan;24(1):51-64.

18. Brodie JS, Di Marzo V, Guy GW. Polypharmacology Shakes Hands with Complex Aetiopathology. Trends in Pharmacological Sciences, Dez. 2015, v.36, n.12, p. 802-821.

19. Devinsky O, Cross JH, Laux L, Marsh E, Miller I, Nabbout $R$, Scheffer IE, Thiele EA, Wright S; Cannabidiol in Dravet Syndrome Study Group. Trial of Cannabidiol for DrugResistant Seizures in the Dravet Syndrome. N Engl ] Med. 2017 May 25;376(21):2011- 2020.

20. Devinsky O, Patel AD, Cross JH, Villanueva V, Wirrell EC, Privitera M, Greenwood SM, Roberts C, Checketts D, VanLandingham KE, Zuberi SM; GWPCARE3 Study Group. Effect of Cannabidiol on Drop Seizures in the Lennox-Gastaut Syndrome. N Engl J Med. 2018 May 17;378(20):1888-1897.

21. Stockings E, Zagic D, Campbell G, Weier M, Hall WD, Nielsen S, Herkes GK, Farrell M, Degenhardt L. Evidence for cannabis and cannabinoids for epilepsy: a systematic review of controlled and observational evidence. J Neurol Neurosurg Psychiatry. 2018 Jul;89(7):741-753.

22. Pamplona FA, da Silva LR, Coan AC. Potential Clinical Benefits of CBD- Rich Cannabis Extracts Over Purified CBD in Treatment-Resistant Epilepsy: Observational Data Meta-analysis. Front Neurol. 2018 Sep 12;9:759

23. Birnbaum AK, Karanam A, Marino SE, Barkley CM, Remmel RP, Roslawski M, Gramling-Aden M, Leppik IE. Food effect on pharmacokinetics of cannabidiol oral capsules in adult patients with refractory epilepsy. Epilepsia. 2019 Aug;60(8):1586-1592.
24. Suraev A, Lintzeris N, Stuart J, Kevin RC, Blackburn R, Richards E, Arnold JC, Ireland C, Todd L, Allsop DJ, McGregor IS. Composition and Use of Cannabis Extracts for Childhood Epilepsy in the Australian Community. Sci Rep. 2018 Jul 5;8(1):10154.

25. O'Connell BK, Gloss D, Devinsky O. Cannabinoids in treatment-resistant epilepsy: A review. Epilepsy Behav. 2017 May;70(Pt B):341-348.

26. Rosenberg EC, Louik J, Conway E, Devinsky O, Friedman D. Quality of Life in Childhood Epilepsy in pediatric patients enrolled in a prospective, open-label clinical study with cannabidiol. Epilepsia. 2017 Aug;58(8):e96-e100.

27. KossoffEH, Dorward JL. The modified Atkins diet. Epilepsia. 2008 Nov;49 Suppl 8:37- 41.

28. Kim DY, Rho JM. The ketogenic diet and epilepsy. Curr Opin Clin Nutr Metab Care. 2008 Mar;11(2):113-20.

29. Masino SA, Rho JM. Mechanisms of Ketogenic Diet Action. In: Noebels JL, Avoli M, Rogawski MA, Olsen RW, DelgadoEscueta AV, editors. Jasper's Basic Mechanisms of the Epilepsies [Internet]. 4th ed. Bethesda (MD): National Center for Biotechnology Information (US); 2012.

30. McNally MA, Hartman AL. Ketone bodies in epilepsy. J Neurochem. 2012 Apr;121 (1): 28-35.

31. Neal EG, Chaffe $H$, Schwartz RH, Lawson MS, Edwards N, Fitzsimmons G, Whitney A, Cross JH. The ketogenic diet for the treatment of childhood epilepsy: a randomised controlled trial. Lancet Neurol. 2008 Jun;7(6):500-6.

32. Kossoff EH, Zupec-Kania BA, Amark PE, Ballaban-Gil KR, Christina Bergqvist AG, Blackford $R$ et al. Optimal clinical management of children receiving the ketogenic diet: recommendations of the International Ketogenic Diet Study Group. Epilepsia. 2009 Feb;50(2):304-17.

33. Kossoff EH, Hartman AL. Ketogenic diets: new advances for metabolism-based therapies. Curr Opin Neurol. 2012 Apr;25(2):173-8.

34. Freeman JM, Kossoff EH, Hartman AL. The ketogenic diet: one decade later. Pediatrics. 2007 Mar;119(3):535-43.

35. Chen W, Kossoff EH. Long-term follow-up of children treated with the modified Atkins diet. ] Child Neurol, 2012, v. 27, n.7, p. 754-8.

36. Milder J, Patel M. Modulation of oxidative stress and mitochondrial function by the ketogenic diet. Epilepsy Res. 2012 Jul;100(3):295-303.

37. Freeman JM, Vining EP, Kossoff EH, Pyzik PL, Ye X, Goodman SN. A blinded, crossover study of the efficacy of the ketogenic diet. Epilepsia. 2009 Feb;50(2):322-5.

38. Lee PR, Kossoff EH. Dietary treatments for epilepsy: management guidelines for the general practitioner. Epilepsy Behav. 2011 Jun;21(2):115-21.

39. Liu YM. Medium-chain triglyceride (MCT) ketogenic therapy. Epilepsia. 2008 Nov;49 Suppl 8:33-6.

40. Kossoff EH, Dorward JL, Molinero MR, Holden KR. The modified Atkins diet: a potential treatment for developing countries. Epilepsia. 2008 Sep;49(9):1646-7.

41. Pfeifer $\mathrm{HH}$, Thiele EA. Low-glycemic-index treatment: a liberalized ketogenic diet for treatment of intractable epilepsy. Neurology. 2005 Dec 13;65(11):1810-2. 
42. Miranda MJ, Turner Z, Magrath G. Alternative diets to the classical ketogenic diet--can we be more liberal? Epilepsy Res. 2012 Jul;100(3):278-85.

43. Kossoff EH, Nabbout R. Use of dietary therapy for status epilepticus. J Child Neurol. 2013 Aug;28(8):1049-51.
44. Stafstrom CE, Rho JM. The ketogenic diet as a treatment paradigm for diverse neurological disorders. Front Pharmacol. 2012 Apr 9;3:59.

45. McNally MA, Pyzik PL, Rubenstein JE, Hamdy RF, Kossoff EH. Empiric use of potassium citrate reduces kidneystone incidence with the ketogenic diet. Pediatrics. 2009 Aug;124(2):e300-4. 